\title{
Evaluación de la adultez emergente en Chile: validación del IDEA - extendido en universitarios chilenos
}

\section{Assessment of emerging adulthood in Chile: Validation of the extended - IDEA in Chilean University Students}

\author{
Ana Barrera-Herrera \\ Departamento de Psicología, Universidad Católica de Temuco, Chile \\ Eugenia V. Vinet \\ Departamento de Psicología, Universidad de La Frontera, Chile \\ Manuel S. Ortiz \\ Departamento de Psicología, Universidad de La Frontera, Chile
}

Rec (23 de enero de 2018) Acept (19 de junio de 2019)

Correspondencia:

Ana Barrera-Herrera, Departamento de Psicología, Universidad Católica de Temuco, Manuel Montt 056, Temuco, Chile. Dirección electrónica: abarrera@uct.cl. Correos de los otros autores: eugenia.vinet@ufrontera.cl, manuel.ortiz@ufrontera.cl.

Notas autor: Este estudio forma parte de la Tesis doctoral de la primera autora, desarrollada en el Programa de Doctorado en Psicología de la Universidad de La Frontera, y recibió financiamiento del Proyecto FONDECYT No 1150095, denominado Adultez Emergente y variables socioculturales y de salud mental en universitarios chilenos. 


\title{
Resumen
}

La adultez emergente es un nuevo periodo evolutivo, que abarca de los 18 a los 29 años. El instrumento más utilizado para evaluarla es el Inventario de Dimensiones de Adultez Emergente (IDEA). El objetivo general del estudio fue evaluar la pertinencia cultural y las características psicométricas del IDEA y de 25 ítems culturales/émicos en una muestra de 589 de universitarios chilenos. Análisis factoriales exploratorios demostraron que el IDEA tiene una estructura similar a la señalada por otro estudio chileno, y los ítems culturales conforman una estructura de tres factores. El análisis factorial confirmatorio arrojó un modelo unifactorial de seis indicadores, tres contienen 21 ítems originales del IDEA, y tres conservan 24 ítems culturales formando un solo instrumento, denominado IDEA - extendido, con propiedades psicométricas adecuadas. Este instrumento caracteriza la adultez emergente como una sola etapa de vida, que contiene aspectos éticos y émicos, dando un panorama integrado de sus características.

Palabras clave: adultez emergente, evaluación, cultura, universitarios

\begin{abstract}
Emerging adulthood is a new evolutionary period ranging from 18 to 29 years. The most used instrument to evaluate it is the Inventory of the Dimensions of Emerging Adulthood (IDEA). The general objective of the study was to evaluate the cultural relevance and psychometric characteristics of the IDEA and 25 cultural/ emic items in a sample of 589 Chilean university students. Exploratory factorial analysis showed that the IDEA has a similar structure to one indicated by another Chilean study, and the cultural items form a threefactor structure. Confirmatory factorial analysis threw a unifactorial model of six indicators, three of them contain 21 original items of the IDEA, and three conserve 24 cultural items forming a single instrument, called extended - IDEA, with adequate psychometric properties. This instrument characterizes the emerging adulthood as a single stage of life, which contains ethical and emic aspects, giving an integrated view of its characteristics.
\end{abstract}

Key words: emerging adulthood, assessment, culture, university students

\section{Introducción}

En los últimos diez años, desde el área de la Psicología Evolutiva, una nueva teoría del desarrollo ha recibido creciente atención. Esta teoría describe el periodo evolutivo situado entre la adolescencia y la adultez, denominado Adultez Emergente (AE), una etapa de vida entre los 18 y los 29 años y cuya característica más relevante es que la mayoría de los jóvenes que transitan por ella no se ven como adolescentes ni tampoco sienten que han alcanzado la adultez (Arnett, 2000, 2004, 2012). Este periodo se manifiesta en sociedades industrializadas a partir de los cambios sociodemográficos de las últimas décadas, tales como el aumento del acceso a la educación universitaria, el aumento de la fuerza laboral y la postergación de hitos como el matrimonio o tener hijos (Arnett, 2000).

De acuerdo con Arnett, Kloep, Hendry \& Tanner (2011), la AE es un periodo único y heterogéneo que presenta características específicas que pueden variar dependiendo de la cultura en la cual se desarrolle el individuo. En Estados Unidos se han descrito cinco características distintivas de esta etapa: (a) Exploración de la identidad; (b) Inestabilidad; (c) gran Optimismo y posibilidades; (d) estar Centrado en sí mismo; y (e) sentirse "En el medio" entre la adolescencia y la adultez (Arnett, 2000, 2004). Para evaluar estas características en los jóvenes estadounidenses, el autor junto a otros investigadores desarrolló el Inventario de Dimensiones de la Adultez Emergente (IDEA; Reifman, Arnett, \& Colwell, 2007). Este inventario ha sido uno de los instrumentos más utilizados para medir la Adultez emergente y cuenta con variados estudios internacionales, los que se presentan a continuación. 


\section{Inventario de Dimensiones de la Adultez Emergente}

Este instrumento tiene como propósito examinar las características distintivas de la AE, además de medir diferencias individuales en la auto-identificación con los procesos específicos de esta etapa de vida. Los ítems que lo componen mapean las cinco dimensiones teóricas de la AE, esto es: Exploración de la identidad, Inestabilidad, Auto-centramiento, Sentirse en medio y Posibilidades (Arnett, 2004). Posteriormente, los autores desarrollaron una dimensión adicional, llamada Centramiento en otros, la cual representa un contrapunto al auto-centramiento.

El IDEA está compuesto por 31 ítems, que comienza con un encabezado en donde se le pide al sujeto que piense en el momento de vida actual y, luego, que indique el grado en el que está de acuerdo o en desacuerdo con cada una de las frases que describe ese periodo de su vida (Reifman et al., 2007). El formato de respuesta es de tipo Likert, el cual considera las siguientes opciones: "Fuertemente en desacuerdo" (1), "En desacuerdo" (2), "De acuerdo" (3) y "Fuertemente de acuerdo" (4). Algunos ejemplos de estos ítems, en su versión en español utilizada en este estudio, son: "Este periodo de mi vida es un tiempo de inestabilidad" (ítem 9), y "Este periodo de mi vida es un tiempo de nuevas posibilidades" (ítem 16).

El IDEA originalmente fue diseñado para jóvenes estadounidenses de 18 a 25 años, y en su complejo proceso de validación, se realizaron cinco estudios de diferentes grupos etarios, estatus educacional y ocupación. De manera complementaria, se analizaron las relaciones entre las dimensiones del instrumento con otras variables psicológicas como satisfacción con la vida, autodominio, búsqueda de novedad, orientación futura e imaginación de posibles sí mismos (Reifman et al., 2007).

Los resultados del proceso de validación muestran como primer hallazgo que las cinco características distintivas de la AE están presentes en el grupo de jóvenes entre 18 y 29 años, puesto que son el grupo que obtiene las medias más altas en las cinco dimensiones, respecto de otros grupos de edad como adolescentes o adultos de mediana edad. Asimismo, psicométricamente, al realizar los distintos análisis factoriales exploratorios y confirmatorios (AFE y AFC), los ítems se agrupan bajo la conceptualización inicial propuesta de AE de cinco subescalas ("Exploración de la Identidad", "Experimentación/Posibilidades", "Negatividad/Inestabilidad", "Autocentrado", y "Sentirse en el medio"); surge además la subescala suplementaria "Centramiento en otros", que revela una estructura final de seis factores con adecuados coeficientes de consistencia interna (Reifman et al., 2007).

\section{Estudios Relevantes del IDEA}

Las investigaciones en torno a este instrumento han seguido desarrollándose internacionalmente a través de distintos estudios empíricos en otras realidades culturales. Con el fin de estudiar el instrumento en otras poblaciones, se realizó un estudio de las propiedades psicométricas de una versión más breve del IDEA(IDEARevisado), compuesto por 21 ítems y aplicado a una muestra de 1.676 jóvenes latinos en riesgo social (Lisha et al., 2012). Los investigadores no incluyeron en la aplicación los ítems de las escalas "Negatividad/Inestabilidad" y "Centrado en otros", debido a que la primera apuntaba a emociones negativas asociadas con la transición hacia la adultez y ya contaban con otras escalas para medir sintomatología asociada a la ansiedad y al estrés. La segunda fue eliminada debido a que era una dimensión complementaria y no formaba parte de la descripción focal de la AE. El análisis factorial de componentes principales de esta muestra arrojó una solución de tres subescalas, denominadas por los autores como: "Exploración de la Identidad", "Experimentación/Posibilidades" e "Independencia", obteniéndose para la escala total un alfa de Cronbach de 0.93.

Más recientemente, un estudio llevado a cabo con una muestra de 2.472 adultos emergentes italianos y japoneses tuvo por objetivo analizar una versión corta del IDEA original (Crocetti et al., 2015). Basándose en la investigación original del IDEA (Reifman et al., 2007), se realizaron las siguientes modificaciones al instrumento: a) se eliminaron los tres ítems que pertenecían a la dimensión complementaria "Centrado en Otros", b) se seleccionaron solo los ítems relacionados conceptualmente con la definición de cada dimensión y que no tuvieran cargas factoriales cruzadas, y c) se mantuvieron los ítems que fueran los mejores indicadores de los factores latentes originales (aquellos con las mayores cargas factoriales reportados en el estudio original del IDEA). Así, se obtuvo un IDEA abreviado de 15 ítems, con tres ítems por cada dimensión. Los AFC muestran un ajuste adecuado del instrumento en ambas muestras. 
En América Latina, en México, Brasil y Chile, también se han realizado estudios que han utilizado el instrumento IDEA. Específicamente, en el año 2007, se realizó un estudio de AE, donde se aplicó el IDEA original en una muestra mixta integrada por 720 mexicanos y españoles entre 16 y 34 años (Fierro \& Moreno, 2007). Los análisis arrojaron una estructura de siete factores para ambos grupos: 1) "Aplazamiento de la adultez", 2) "Inestabilidad", 3) "Autonomía"; 4) "Exploraciones", 5) "Visión de futuro y posibilidades", 6) "Preocupaciones", y 7) "Moratoria de Identidad". Para la escala total, se obtuvo una confiabilidad en ambas muestras de 0.68 .

En tanto, en Brasil, se realizó una adaptación del IDEA original (Dutra-Thomé, 2013). Previo a su aplicación, se agregaron dos ítems al conjunto original de 31 reactivos, a fin de representar de mejor manera a la muestra brasileña: "Tiempo para prepararte para la adultez", y "Tiempo para decisiones profesionales". El análisis factorial de ejes principales arrojó un instrumento compuesto por 29 ítems, que forman una estructura factorial muy similar a las seis dimensiones originales del instrumento: 1) "Exploración de la Identidad", 2) "Experimentación/Posibilidades", 3) "Negatividad/Inestabilidad", 4) "Autocentrado", 5) "Sentirse en el medio", y 6) "Centrado en otros". La confiabilidad para la escala total fue de un valor de 0.80 .

En el año 2008, se realizó una investigación del IDEA en una muestra de jóvenes chilenos (IDEA PUC; Pérez, Cumsille, \& Martínez, 2008), cuyo objetivo fue analizar la validez de constructo de este inventario. Los investigadores tradujeron al español el instrumento original y fue aplicado a una muestra de 192 adultos jóvenes voluntarios, de 18 a 26 años de edad, de los cuales un $91 \%$ de la muestra eran estudiantes universitarios con una edad promedio de 20 años.

El AFE realizado arrojó una estructura de cuatro factores, dos de los cuales son consistentes con la solución factorial original del IDEA (Factor 2 y 3 ). Estas cuatro dimensiones se denominaron de la siguiente manera: 1) "Exploración de la identidad", que agrupa ítems de la dimensión original del mismo nombre y de la dimensión original de "Sentirse en el medio"; (2) "Libertad/posibilidades", que incluye los ítems de la dimensión original de "Experimentación/Posibilidades"; (3) "Negatividad/Inestabilidad", la cual mantiene en su mayoría sus ítems originales; y (4) "Sí mismo/Otros", que abarca ítems relacionados con las dimensiones originales de "Autocentramiento" y "Centrarse en otros". No se reportan las confiabilidades de cada factor, y se decide mantener la estructura original de 31 ítems (Pérez et al., 2008).

Como se observa, el instrumento IDEA ha sido estudiado en distintas realidades culturales, donde han surgido estructuras factoriales distintas respecto del instrumento original. En estas investigaciones, se ha modificado la extensión del IDEA y ha evolucionado hacia un instrumento más breve o más extenso, que en general tiene propiedades psicométricas adecuadas. En el ámbito nacional, la estructura de cuatro factores obtenida en el estudio chileno, también es diferente de la subyacente al IDEA original. Esta falta de replicabilidad de la estructura factorial original del IDEA, en los distintos estudios descritos, constituye un antecedente importante, pues hace suponer que, la forma en que se desarrolla esta etapa podría tener características culturales distintas a las descritas en la cultura norteamericana, lo que podría explicar esta diversidad de estructuras factoriales obtenidas.

$\mathrm{Al}$ evaluar la $\mathrm{AE}$ con un instrumento, es importante considerar que las culturas son entidades complejas y variables, donde coexisten aspectos éticos (características y significados universales) y émicos (características, significados y conceptos particulares de un determinado contexto cultural, que tienen sentido sólo en ese marco de referencia), aspectos que deben estar contenidos en el instrumento de evaluación (Berry, Poortinga, Segall, \& Dasen, 1992).

Estos antecedentes sugieren la necesidad de realizar una adaptación cultural del IDEA en Chile, estudiar las características psicométricas de los ítems originales y disponer de ítems culturales o émicos que den cuenta de las características particulares de esta etapa de vida en jóvenes chilenos, a fin de tener un instrumento de medición que cuente con una equivalencia lingüística, cultural, conceptual y métrica (Muñiz, Elosua, \& Hambleton, 2013) del constructo de AE de la realidad local chilena.

Por lo tanto, el objetivo general de este estudio psicométrico fue evaluar la pertinencia cultural y las características psicométricas del Inventario de Dimensiones de Adultez Emergente (Reifman et al., 2007), y de los ítems émicos derivados de una etapa previa de investigación (Barrera-Herrera, 2016) en una muestra de universitarios chilenos.

Las hipótesis vinculadas a esta etapa son las siguientes:

H1. El IDEA original se estructurará de forma similar a las dimensiones descritas por el estudio del IDEA PUC (Pérez et al., 2008). 
H2. Los ítems émicos se estructurarán en forma independiente, pero relacionados con las dimensiones de los ítems originales, de manera que complementen la caracterización general de AE.

No se formulan hipótesis específicas respecto de la naturaleza de la relación entre los dos grupos de ítems, ya que no existen antecedentes previos.

\section{Método}

Se utilizó un diseño no experimental, transversal, con un alcance descriptivo y correlacional. No fue experimental, pues no existió manipulación de variables; la medición se realizó en un momento determinado y los objetivos estuvieron centrados en describir y caracterizar la AE chilena, en términos de las dimensiones obtenidas.

\section{Participantes}

Mediante un muestreo no probabilístico, por conveniencia, se evaluó a una muestra nacional de 589 universitarios, pertenecientes a diversas carreras de universidades estatales de tres ciudades chilenas, Santiago, Talca y Temuco. La descripción de esta muestra se presenta en detalle en la sección correspondiente de los resultados. Los criterios de inclusión fueron: a) estar en tercero, cuarto o quinto año de una carrera universitaria, b) ser chileno/a, y c) tener entre 19 y 29 años.

\section{Procedimiento}

Los participantes fueron contactados a través de sus profesores o directores de carrera, quienes autorizaron realizar la aplicación de la encuesta durante el periodo de clases. Antes de realizar la aplicación, se explicó a los alumnos, de forma breve, el propósito de la investigación y se destacó el carácter voluntario de su participación. A aquellos que accedieron a participar se les entregó el consentimiento informado visado por el Comité Ético Científico de la Universidad de La Frontera y, luego de firmar, se procedió a la aplicación del instrumento en un período de 40 minutos aproximadamente.

\section{Instrumentos}

Los universitarios contestaron una encuesta, que incluía lo siguiente: en primer lugar, se adjuntó un Cuestionario sociodemográfico, el cual es un instrumento de autorreporte que incluyó variables para caracterizar la muestra, tales como edad, sexo, carrera, nivel socioeconómico, entre otras. En segundo lugar, se incluyó el Inventario de Dimensiones de Adultez Emergente (IDEA PUC), utilizando la versión chilena del IDEA (Pérez et al., 2008), la cual fue ajustada por el equipo de investigación en términos de lenguaje y redacción. En tercer lugar, se incluyeron los Ítems émicos/culturales de la AE en Chile, el cual es un conjunto de 25 ítems, surgidos de una etapa previa de investigación, los cuales abordan las características centrales de la AE en Chile, específicamente en temáticas como el área Interpersonal, Relaciones Familiares, y Amor y Sexualidad.

\section{Análisis}

Las respuestas de la encuesta de los 589 participantes fueron traspasadas a una base de datos en el programa estadístico SPSS 21.0, en la cual se codificaron preguntas abiertas del cuestionario sociodemográfico y se examinó la presencia de datos perdidos.

A continuación, se realizó una serie de análisis descriptivos que incluyeron: caracterización sociodemográfica de la muestra, cómputo de las escalas del IDEA en su versión original y cómputo de las escalas según la solución factorial obtenida por el estudio del IDEA PUC (Pérez et al., 2008). Para cada una de las escalas computadas, se determinó también su consistencia interna a través del coeficiente Alfa de Cronbach.

Luego, mediante un procedimiento al azar, la muestra total fue dividida en dos. Un $50 \%$ de 10 casos $(n=297)$ fue destinado para realizar análisis factoriales exploratorios (AFE; Muestra 1), en tanto, el 50\% restante fue utilizado en los análisis factoriales confirmatorios (AFC; Muestra 2). 
Con la muestra 1, se realizó el examen de la matriz de correlaciones bivariadas y de los índices de KMO y la prueba de esfericidad de Bartlett, tanto para los ítems del IDEA original como para el conjunto de los 25 ítems émicos creados por el equipo. A continuación, se realizaron distintos AFE para los dos grupos de ítems por separado, los cuales fueron evaluados según su pertinencia teórica y sus indicadores psicométricos. Las estructuras factoriales con mejor ajuste teórico y psicométrico fueron llevadas a AFC desarrollados con la muestra 2, los cuales también fueron examinados según criterios teóricos y psicométricos.

En la muestra 2, debido al no cumplimiento del supuesto de normalidad multivariada (Mardias multivariate test $=168.22, \mathrm{p} \leq 0.001$ ), se realizaron tres AFC con indicadores robustos (Satorra-Bentler), que corrigen el no cumplimiento del supuesto. Los AFC se realizaron con una estimación de máxima verosimilitud (ML) y se utilizaron los siguientes indicadores de bondad de ajuste, cuyos valores de referencia han sido propuestos por Hu \& Bentler (1999):

- Chi-cuadrado $\left(\chi^{2}\right)$, Índice de Ajuste Comparativo (CFI mayor o igual a 0.95),

- Índice de Tucker Lewis (TLI mayor o igual a 0.90),

- Error de Aproximación Cuadrático Medio (RMSEA menor o igual 0.06), y el

- Promedio de los Residuales Estandarizados (SRMR menor o igual a 0.08).

Finalmente, para estos análisis se utilizó el software STATA 14.0 MP.

\section{Resultados}

\section{Descripción de la Muestra}

La muestra estuvo compuesta por 589 sujetos. Un $58.4 \%$ de los participantes eran mujeres y la media de edad fue de 21.13 años con una DT de 2.60. Los participantes, en su mayoría, provenían de centros urbanos medianos y grandes, con más de 15.000 habitantes (69.9\%). Más de la mitad de los universitarios vivía con sus padres (64.5\%) y los que vivían de forma independiente, con amigos o familiares, lo hacían por motivos de estudios. En relación con el estado civil, la mayor parte de la muestra eran solteros (56.5\%), un 43\% declaró estar en una relación de pareja y el $95.9 \%$ no tenía hijos.

En cuanto a la pertenencia étnica, un $91.9 \%$ se declaró como chileno y un $6.1 \%$ se adscribía a la etnia mapuche. Respecto del nivel socioeconómico de los participantes, la mayor parte de éstos pertenecía al nivel medio (31.2\%), seguido por el nivel medio-alto (25.5\%) y por el nivel medio-bajo (24.5\%).

En relación con otras variables, como el trabajo, la mayor parte de los adultos emergentes universitarios de esta muestra no trabajaba (78.4\%), y los que sí, laboraban entre 8 y 10 horas semanales. Asimismo, en cuanto a la variable religión, un $63.9 \%$ señaló no practicar ninguna religión y un $36.1 \%$ afirmo qué sí, principalmente la religión católica ( $21 \%)$ o evangélica $(13.9 \%)$.

\section{Análisis Factoriales Exploratorios}

Con la muestra 1, se estimó el índice de adecuación muestral (KMO) y el supuesto de esfericidad de Bartlett, tanto para los 31 ítems originales del IDEA como para el conjunto de 25 ítems émicos. Para el primer conjunto de datos, el KMO obtenido $(\mathrm{KMO}=0.81)$ es considerado un valor bueno, y la prueba de esfericidad resultó ser estadísticamente significativa $\left(X^{2}{ }_{(465)=} 2963.18 ; p \leq 0.001\right)$; además, se obtuvo un alfa de Cronbach de 0.79. Por su parte, para el conjunto de 25 ítems creados por el equipo de investigación, el KMO obtenido (0.85) es adecuado, la prueba de esfericidad resultó ser estadísticamente significativa $\left(X_{(300)}^{2}=2558.21 ; p \leq 0.001\right)$, y se obtuvo un alfa de 0.87 considerando este set de ítems. Estos valores indican que ambos conjuntos de datos son factorizables y permiten continuar con los análisis. 
Tabla 1. Estadísticos Descriptivos de variables sociodemográficas

\begin{tabular}{|c|c|c|c|}
\hline Variable & & $\mathbf{N}$ & $\%$ \\
\hline \multirow[t]{2}{*}{ Sexo } & Masculino & 245 & 41.6 \\
\hline & Femenino & 344 & 58.4 \\
\hline \multirow[t]{4}{*}{ Región de Procedencia } & $\mathrm{RM}$ & 169 & 28.5 \\
\hline & Maule & 162 & 27.3 \\
\hline & Araucanía & 144 & 24.2 \\
\hline & Otras & 119 & 20.0 \\
\hline \multirow[t]{2}{*}{ Vives con } & Uno o ambos padres & 291 & 64.0 \\
\hline & Independiente de los padres & 211 & 35.5 \\
\hline \multirow[t]{3}{*}{ Estado Civil } & Soltero(a) & 335 & 56.5 \\
\hline & En pareja & 255 & 43.0 \\
\hline & Casado & 1 & 0.2 \\
\hline \multirow[t]{2}{*}{ Hijos } & No & 564 & 95.9 \\
\hline & Sí & 24 & 4.1 \\
\hline \multirow[t]{3}{*}{ Universidad } & Talca & 199 & 33.5 \\
\hline & Temuco & 198 & 33.3 \\
\hline & Santiago & 197 & 33.2 \\
\hline \multirow[t]{3}{*}{ Etnia } & Chileno & 545 & 91.9 \\
\hline & Mapuche & 36 & 6.1 \\
\hline & Otra & 12 & 0.2 \\
\hline \multirow[t]{2}{*}{ Trabajo } & No & 457 & 78.4 \\
\hline & Sí & 126 & 21.4 \\
\hline \multirow[t]{4}{*}{ Practica Religión } & No & 377 & 63.9 \\
\hline & Católico & 124 & 21.0 \\
\hline & Evangélico & 82 & 13.9 \\
\hline & Otro & 7 & 1.2 \\
\hline \multirow{2}{*}{$\begin{array}{l}1^{\circ} \text { Generación } \\
\text { Universidad }\end{array}$} & No & 382 & 64.3 \\
\hline & Sí & 212 & 35.7 \\
\hline \multirow[t]{6}{*}{ Nivel Socioeconómico } & NSE bajo & 24 & 4.1 \\
\hline & NSE Medio bajo & 143 & 24.5 \\
\hline & NSE Medio & 182 & 31.2 \\
\hline & NSE Medio Alto & 149 & 25.5 \\
\hline & NSE Alto & 57 & 9.8 \\
\hline & NSE Muy Alto & 29 & 5.0 \\
\hline
\end{tabular}

Fuente: Elaboración propia.

\section{AFE del IDEA original.}

Con los 31 ítems originales del IDEA, se realizaron cuatro análisis: 1) AFE para indagar cómo se distribuyen los ítems sin forzar ningún factor; 2) AFE forzando una solución de seis factores, para observar si la distribución obtenida se asemeja a la propuesta de seis factores del IDEA original; 3) AFE forzando una solución de cuatro factores, a fin de observar si la distribución obtenida empíricamente es similar a la propuesta por el estudio del IDEA PUC; y 4) AFE forzando una solución de cuatro factores, donde se eliminan los ítems con 
baja carga factorial, con el objeto de tener una solución final para llevar al AFC. Todos los AFE realizados fueron ejecutados con extracción de componentes principales, rotación oblimin, y considerando los ítems con carga factorial sobre 0.30 .

Los dos primeros AFE ( 1 y 2 ) resultaron insatisfactorios, debido a la gran cantidad de factores obtenidos y porque la estructura original de seis factores no aparece a partir del conjunto de datos sometido a este análisis, por lo tanto, se descartan las soluciones obtenidas por sus debilidades psicométricas y sólo se reportan los AFE restantes.

En el tercer análisis, se realizó un AFE forzando una solución de cuatro factores, con el fin de probar la estructura chilena del IDEA PUC. En este análisis la estructura empírica obtenida se asemeja y casi se replica la estructura de cuatro factores propuesta por el estudio del IDEA PUC, lo que confirma la hipótesis 1 que señala que el IDEA original se estructuraría de forma similar a la obtenida en el estudio del IDEA PUC. En segundo lugar, tres de sus dimensiones presentan adecuada confiabilidad (F1, F2, F3, con valores alfa de $0.81,0.80$ y 0.77 respectivamente), y la mayoría de los ítems posee una carga factorial adecuada y buena correlación ítem-test.

Sin embargo, se observan algunas anomalías, por ejemplo, el tercer factor presenta problemas en el ítem 17 con una baja correlación ítem-total, menor a 0.30. Además, el cuarto factor presenta ciertas dificultades con tres ítems $(14,22$ y 23$)$, que no se correlacionan adecuadamente con los otros ítems del factor, ya que presentan valores menores a 0.30 ; sin embargo, al calcular la confiabilidad de este factor eliminando los ítems problemáticos, la confiabilidad se mantiene en el valor de 0.68. Estos resultados evidencian algunos problemas en esta estructuración de cuatro factores pese a ser más adecuada que la anterior de seis factores.

En el cuarto análisis, se forzó una solución de cuatro factores, eliminando los ítems problemáticos aparecidos en los análisis previos (ítems 14, 17, 22, y 23). En la Tabla 2, se presenta la estructura final de cuatro factores, que contiene los 27 ítems rescatados del conjunto de ítems del IDEA original, la cual es factible de ser comprobada en un AFC. Estos cuatro factores se denominan de acuerdo con su contenido teórico de la siguiente forma: F1: Apertura personal, F2: Presión y estrés, F3: Autosuficiencia, y F4: Construcción de la identidad.

Tabla 2. AFE de 27 items originales del IDEA donde se forzó una solución de cuatro factores en la muestra 1

\begin{tabular}{llll}
\hline Factor & Ítem & Carga factorial & $\begin{array}{l}\text { Correlación Ítem } \\
\text { - Total }\end{array}$ \\
\hline F1 & & & 0.57 \\
Apertura personal & 1. Tiempo de muchas posibilidades & 0.68 & 0.59 \\
2. Factor 1: 0.81 & 4. Tiempo de exploración & 0.74 & 0.49 \\
& 5. Tiempo de libertad personal & 0.68 & 0.54 \\
& 10. Tiempo de optimismo & 0.60 & 0.51 \\
& 16. Tiempo de nuevas posibilidades & 0.58 & 0.61 \\
& 21. Tiempo para probar cosas nuevas & 0.60 & 0.53 \\
\hline F2 & 3. Tiempo de confusión & 0.60 & 0.53 \\
Presión y estrés & 6. Tiempo de sentirse restringido(a) & 0.61 & 0.37 \\
$\alpha$ Factor 2: 0.77 & 8. Tiempo de sentirse estresado(a) & 0.39 & 0.65 \\
& 9. Tiempo de inestabilidad & 0.82 & 0.51 \\
& 11. Tiempo de alta presión & 0.57 & 0.51 \\
& 20. Tiempo de muchas preocupaciones & 0.81 & 0.56 \\
\hline
\end{tabular}




\begin{tabular}{|c|c|c|c|c|c|}
\hline Factor & \multicolumn{3}{|l|}{ Ítem } & Carga factorial & $\begin{array}{l}\text { Correlación Ítem } \\
\text { - Total }\end{array}$ \\
\hline \multirow{2}{*}{$\begin{array}{l}\text { F3 } \\
\text { Autosuficiencia }\end{array}$} & \multicolumn{3}{|c|}{ 7. Tiempo de responsabilidad contigo mismo(a) } & 0.61 & 0.37 \\
\hline & \multicolumn{3}{|c|}{ 13. Tiempo para establecerse } & 0.61 & 0.45 \\
\hline \multirow{4}{*}{$\alpha$ Factor 3: 0.68} & \multicolumn{3}{|c|}{ 15. Tiempo de independencia } & 0.46 & 0.38 \\
\hline & \multicolumn{3}{|c|}{ 18. Tiempo para comprometerse con otros } & 0.50 & 0.39 \\
\hline & \multicolumn{3}{|c|}{ 19. Tiempo para ser autosuficiente } & 0.68 & 0.46 \\
\hline & \multicolumn{3}{|c|}{ 25. Tiempo para planear el futuro } & 0.54 & 0.40 \\
\hline \multirow{3}{*}{$\begin{array}{l}\mathrm{F} 4 \\
\text { Construcción de la } \\
\text { identidad }\end{array}$} & \multicolumn{3}{|c|}{ 12. Tiempo para descubrir quién eres } & -0.64 & 0.57 \\
\hline & \multicolumn{3}{|c|}{ 24. Tiempo para definirte a ti mismo(a) } & -0.60 & 0.56 \\
\hline & \multicolumn{3}{|c|}{ 26. Tiempo de búsqueda de significados } & -0.64 & 0.51 \\
\hline \multirow[t]{5}{*}{$\alpha$ Factor 4: 0.80} & \multicolumn{3}{|c|}{ 27. Tiempo para decidir tus creencias y valores } & -0.77 & 0.62 \\
\hline & \multicolumn{3}{|c|}{ 28. Tiempo para aprender a pensar por ti mismo(a) } & -0.79 & 0.63 \\
\hline & \multicolumn{3}{|c|}{$\begin{array}{l}\text { 29. Tiempo de sentirte adulto en algunas cosas pero no } \\
\text { en otras }\end{array}$} & -0.50 & 0.39 \\
\hline & \multicolumn{3}{|c|}{ 30. Tiempo para convertirse gradualmente en adulto } & -0.51 & 0.45 \\
\hline & \multicolumn{3}{|c|}{$\begin{array}{l}\text { 31. Tiempo de no estar seguro(a) si has alcanzado la } \\
\text { plena adultez }\end{array}$} & -0.50 & 0.40 \\
\hline \multicolumn{2}{|c|}{ Correlación de componentes } & F1 & $\mathrm{F} 2$ & F3 & $\mathrm{F} 4$ \\
\hline \multicolumn{2}{|l|}{ F1 Apertura personal } & - & -0.19 & 0.22 & -0.23 \\
\hline \multicolumn{2}{|l|}{ F2 Presión y estrés } & - & - & -0.16 & -0.17 \\
\hline \multicolumn{2}{|l|}{ F3Autosuficiencia } & - & - & - & -0.12 \\
\hline \multicolumn{2}{|c|}{ F4 Construcción de identidad } & - & - & - & - \\
\hline
\end{tabular}

Nota: $\left(\mathrm{KMO}=0,82 ; X_{(351)}=2625.06 ; p \leq 0.001\right)$

Fuente: Elaboración propia.

\section{AFE de los 25 items émicos.}

Para este conjunto de datos, se realizaron dos análisis: AFE para analizar cómo se distribuyen los 25 ítems émicos, sin forzar ningún factor y AFE de los ítems émicos forzando una solución de tres factores.

En el primer AFE, se obtiene una estructura de seis factores, la cual no resulta satisfactoria, pues la agrupación de ítems no tiene sentido teórico, ya que los ítems que integran los factores evalúan contenidos muy diferentes. Además, 24 de un total de 25 ítems carga en las tres primeras dimensiones y quedan las últimas tres dimensiones compuestas por ítems con bajas cargas factoriales o un solo ítem, lo que afecta de forma drástica su confiabilidad. Por estas razones, se decide descartar esta solución inicial de seis factores.

En el segundo AFE, inicialmente, los ítems émicos surgieron de tres tópicos investigados en etapas previas, por lo tanto, se fuerza una solución de tres factores y se entrega una solución más parsimoniosa en cuanto al contenido de los ítems en cada factor, pues se agrupan ítems que tienen contenido similar. Los dos primeros factores poseen mayor consistencia interna en cuanto a similitud de los contenidos de los ítems, además de una adecuada confiabilidad ( $\left.\mathrm{F} 1_{\text {alfa }}=0.84, y \mathrm{~F} 2_{\text {alfa }}=0.81\right)$. El tercer factor tiene una confiabilidad más baja $(\alpha=0.66)$, ya que contiene un ítem problemático (ítem 40 "Tiempo de sentirse entre la adolescencia y la adultez") con un valor inferior a 0.30 en la correlación ítem total. La confiabilidad de este factor se mantiene en 0.69 y el ítem 40 es eliminado. La solución final para este grupo de ítems, donde se elimina el ítem 40, se presenta en la Tabla 3. 
Tabla 3. AFE de la distribución de 24 ítems émicos, que fuerza una solución de tres factores en la muestra 1

\begin{tabular}{|c|c|c|c|}
\hline Factor & Ítem & Carga factorial & $\begin{array}{l}\text { Correlación Ítem } \\
\text { - Total }\end{array}$ \\
\hline \multirow{9}{*}{$\begin{array}{l}\text { F1 } \\
\text { Relaciones } \\
\text { interpersonales }\end{array}$} & 43. Tiempo para establecer nuevos vínculos con otros & 0.46 & 0.54 \\
\hline & $\begin{array}{l}\text { 44. Tiempo para conocer a una gran diversidad de } \\
\text { personas }\end{array}$ & 0.58 & 0.60 \\
\hline & $\begin{array}{l}\text { 46. Tiempo para tener relaciones de pareja sin mayor } \\
\text { compromiso }\end{array}$ & 0.57 & 0.39 \\
\hline & 48. Tiempo de exploración en la sexualidad & 0.62 & 0.52 \\
\hline & 49. Tiempo para divertirse & 0.76 & 0.63 \\
\hline & 50. Tiempo de prueba en las relaciones de pareja & 0.55 & 0.49 \\
\hline & 51. Tiempo de participación social y/o ciudadana & 0.54 & 0.53 \\
\hline & 52. Tiempo para una sexualidad más activa & 0.59 & 0.47 \\
\hline & $\begin{array}{l}\text { 53. Tiempo de desarrollo de habilidades personales y } \\
\text { sociales }\end{array}$ & 0.49 & 0.52 \\
\hline \multirow{2}{*}{$\alpha$ Factor 1: 0.84} & 55. Tiempo de libertad para equivocarse & 0.49 & 0.47 \\
\hline & 56. Tiempo para pasarlo bien & 0.77 & 0.59 \\
\hline \multirow[t]{6}{*}{$\begin{array}{l}\text { F2 } \\
\text { Autonomía }\end{array}$} & $\begin{array}{l}\text { 35. Tiempo para tomar decisiones personales } \\
\text { importantes }\end{array}$ & -0.63 & 0.49 \\
\hline & 36. Tiempo para lograr autonomía & -0.83 & 0.67 \\
\hline & 37. Tiempo de consolidación de la propia identidad & -0.51 & 0.53 \\
\hline & $\begin{array}{l}\text { 38. Tiempo para afrontar nuevas responsabilidades } \\
\text { personales }\end{array}$ & -0.71 & 0.61 \\
\hline & 39. Tiempo para hacerse cargo de sí mismo(a) & -0.82 & 0.58 \\
\hline & $\begin{array}{l}\text { 42. Tiempo de una relación más autónoma con los } \\
\text { padres }\end{array}$ & -0.44 & 0.42 \\
\hline \multirow[t]{2}{*}{$\alpha$ Factor 2: 0.81} & $\begin{array}{l}\text { 45. Tiempo para independizarse progresivamente de los } \\
\text { padres }\end{array}$ & -0.54 & 0.50 \\
\hline & 47. Tiempo para plantearse metas propias & -0.49 & 0.45 \\
\hline \multirow{4}{*}{$\begin{array}{l}\mathrm{F} 3 \\
\text { Reflexión }\end{array}$} & 32. Tiempo de exposición a la diversidad & 0.47 & 0.36 \\
\hline & $\begin{array}{l}\text { 33. Tiempo para clarificar la elección vocacional y/o } \\
\text { laboral }\end{array}$ & 0.64 & 0.45 \\
\hline & 34. Tiempo para aprender a autorregularse & 0.46 & 0.46 \\
\hline & 41. Tiempo de reflexión & 0.54 & 0.48 \\
\hline$\alpha$ Factor 3: 0.66 & $\begin{array}{l}\text { 54. Tiempo de cuestionamientos en muchas áreas de la } \\
\text { vida }\end{array}$ & 0.69 & 0.34 \\
\hline $\begin{array}{l}\text { Correlación de } \\
\text { componentes }\end{array}$ & F2 & F3 & \\
\hline $\begin{array}{l}\text { F1 Relaciones } \\
\text { interpersonales }\end{array}$ & -0.28 & 0.22 & \\
\hline F2 Autonomía & -- & -0.27 & \\
\hline F3 Reflexión & -- & -- & \\
\hline
\end{tabular}

Nota: $\left(\mathrm{KMO}=0.85 ; X_{(276)}^{2}=2515.92 ; p \leq 0.001\right)$

Fuente: Elaboración propia. 
Considerando los contenidos agrupados, la primera dimensión ha sido llamada "Relaciones interpersonales", la segunda, "Autonomía" y la tercera, "Reflexión". Esta solución empírica de tres factores muestra que los ítems émicos conforman tres dimensiones con contenidos, en su mayoría no cubiertos por el IDEA original, que dan cuenta de características de la $\mathrm{AE}$ en Chile. A su vez, existen dimensiones que agrupan contenidos similares como las dimensiones de Autonomía (contenido émico) y Autosuficiencia (contenido ético). Estos resultados empíricos afirman la hipótesis 2 , que señala que los ítems émicos se estructurarán en forma independiente, pero relacionados con las dimensiones previas del IDEA original. Esta estructura de tres factores agrupa 24 ítems émicos que tienen una adecuada pertinencia de contenido y empírica, y es factible de ser analizada a través de un AFC posteriormente.

\section{Análisis Factoriales Confirmatorios}

Tres modelos empíricos fueron sometidos a un análisis factorial confirmatorio (AFC), donde se buscaba probar las estructuras factoriales derivadas de los análisis exploratorios, esto es la estructura de cuatro factores de los ítems del IDEA original y la de tres factores correspondiente a los ítems émicos. El modelo 1 incluyó dos factores latentes correlacionados denominados Factor Ético (Apertura personal, Presión y estrés, Autosuficiencia y Construcción de la identidad), y Factor Émico (Relaciones interpersonales, Autonomía y Reflexión). En el modelo 2 se probó un modelo similar al anterior, esto es, los Factores Ético y Émico correlacionados, pero esta vez se eliminó el indicador "Presión y estrés" en el Factor Ético, por sus debilidades estadísticas presentadas en el modelo 1. Ahora bien, ambos modelos fueron descartados debido a que, por un lado, el modelo 1 obtuvo pobres indicadores de bondad de ajuste (Ver tabla 4), una alta correlación entre factores y débil carga factorial del indicador "Presión y estrés"; por otro lado, el modelo 2 obtuvo indicadores RMSEA y TLI fuera del rango esperado y alta correlación entre ambos factores, por tanto, solo se informa en detalle el tercer modelo.

En el Modelo 3, se decidió realizar un nuevo AFC y probar, esta vez, una estructura compuesta por un solo factor latente denominado IDEA - extendido, el cual reúne los tres indicadores éticos y los tres émicos, lo que resultó ser una versión más extensa y continua del constructo de AE. Para este modelo, los indicadores robustos de bondad de ajuste fueron: $\chi_{(6)}^{2}=14.32, p \leq 0.05 ; \mathrm{CFI}=0.98$, TLI $=0.95$; el RMSEA fue 0.07; y el SRMR fue de 0.035 . Las cargas factoriales de todos los indicadores se ubican en el rango de 0.46 a 0.54 .

Tabla 4. Indicadores de bondad de ajuste de los tres modelos analizados en los AFC

\begin{tabular}{llllll}
\hline Modelos & $\chi^{2}$ & CFI & TLI & RMSEA & SRMR \\
\hline Modelo 1 & $\chi_{(13)}^{2}=158.02$ & 0.73 & 0.56 & 0.19 & 0.09 \\
& $p \leq 0.001$ & & & & \\
Modelo 2 & $\chi_{(6)}^{2}=25.91$ & 0.95 & 0.88 & 0.10 & 0.44 \\
& $p \leq 0.001$ & & & & \\
Modelo 3 & $\begin{array}{l}\chi_{(6)}^{2}=14.32 \\
p \leq 0.005\end{array}$ & 0.98 & 0.95 & 0.07 & 0.03 \\
& & & & \\
\hline
\end{tabular}

Fuente: Elaboración propia.

A fin de mejorar este modelo, se han incluido tres tipos de covarianza de errores, cuyos valores son pequeños, según lo esperado desde el AFC. La primera covarianza entre los indicadores de "Autosuficiencia" y "Relaciones interpersonales" tiene un valor negativo de -0.52 ; la segunda, entre los indicadores de "Construcción de la identidad" y "Reflexión" tiene un valor de 0.50; y la tercera, entre los indicadores de "Autonomía" y "Reflexión" tiene un valor de 0.20 .

Este modelo posee indicadores adecuados de ajuste y es un modelo más parsimonioso que los anteriores, pues abarca en un solo factor los seis indicadores. Por estas razones, se seleccionó este modelo como la solución final en este estudio.

A fin de complementar el AFC anterior, se calcularon los estadísticos de consistencia interna de esta solución. 
Para la escala global, el valor Alfa de Cronbach obtenido es 0.89; para las seis subescalas del IDEA - extendido los valores Alfa se sitúan entre 0.60 y 0.79: Apertura personal (0.78), Autosuficiencia (0.60), Construcción de la identidad (0.74), Relaciones interpersonales (0.79), Autonomía (0.77) y Reflexión (0.64), los cuales son, en general, adecuados niveles de consistencia interna para cada una de las seis escalas que componen el IDEA - extendido.



Figura 1. AFC con modelo 3 compuesto por un factor latente y seis factores en la muestra 2

\section{Discusión y Conclusiones}

Asumiendo el desafío de desarrollar un instrumento de AE con equivalencia cultural, conceptual y métrica, el objetivo del estudio fue evaluar la pertinencia cultural y las características psicométricas de un instrumento foráneo de la adultez emergente (IDEA; Reifman et al., 2007) y de los ítems émicos derivados de etapas previas de investigación, en una muestra nacional de universitarios chilenos.

Dentro de los principales hallazgos, se confirmó la hipótesis 1 referente a que el IDEA original de 31 ítems (Reifman et al., 2007) se estructuraría de forma similar a las cuatro dimensiones del IDEA PUC (Pérez et al., 2008). La confirmación de esta hipótesis era esperable, pues el estudio del IDEA PUC fue realizado mayoritariamente con una muestra universitaria de características sociodemográficas similares a la muestra de este estudio. No se esperaba que los ítems originales obtuvieran la estructura de seis dimensiones informadas por Reifman et al. (2007), pues la revisión acabada de los estudios previos del IDEA demostró que la estructura de seis factores 
del instrumento original ha sido difícilmente replicada en otras realidades culturales, lo que hace suponer que las características culturales de la AE pueden tener influencia en las distintas estructuras factoriales obtenidas.

Pese a esta similitud de cuatro factores, obtenida entre el presente estudio y el IDEA PUC, fue necesario hacer ajustes y realizar un nuevo AFE eliminando cuatro ítems que tenían una carga factorial bajo 0.30 (ítems 14: "Tiempo para responsabilizarse por otros", 17: "Tiempo impredecible", 22: "Tiempo para centrarte en ti mismo(a)" y 23: "Tiempo para separarte de tus padres"). Desde un punto de vista teórico, estos contenidos pudieron ser cuestionados por los jóvenes, pues hacían referencia a responsabilidades con otros y con ellos mismos, los que son aspectos rechazados por estar asociados a la adultez (ítem 14 y 22), o por la ansiedad que implica alejarse de los padres (23), o la falta de contextualización del ítem 17. Así, se obtuvo una estructura de cuatro factores, donde se mantuvieron 27 ítems del IDEA original, de propiedades psicométricas adecuadas y que luego fue sometida a AFC. Estos cuatro factores fueron denominados: "Apertura personal", "Presión y estrés", "Autosuficiencia" y "Construcción de la identidad". Si bien estas denominaciones son nuevas, es importante señalar que se mantienen en las dimensiones los contenidos de Identidad, Inestabilidad, Autocentramiento y Exploración, del instrumento original. Sin embargo, como existe una nueva organización de ítems, se optó por denominar estas dimensiones con base en los contenidos más presentes en éstas, que tuvieran un peso factorial importante y que fueran términos más contextualizados y cercanos para los jóvenes.

Otro hallazgo importante en relación con la hipótesis 2 consiste en que se confirma que los ítems émicos/ culturales se estructuran de forma independiente, pero están relacionados con las dimensiones de los ítems originales. Los AFE del conjunto de 25 ítems émicos arrojaron una estructura factorial de tres factores, con adecuada confiabilidad. De este conjunto, solo un ítem fue eliminado (ítem 40: "Tiempo de sentirse entre la adolescencia y la adultez") por su baja carga factorial. Estas tres dimensiones obtenidas fueron denominadas "Relaciones interpersonales", "Autonomía" y "Reflexión". Las nuevas dimensiones constituyen dimensiones independientes; sin embargo, están relacionadas entre ellas debido a que dan cuenta de las características culturales de la AE chilena y, si bien algunas dimensiones se asimilan en su denominación con las dimensiones originales (tales como "Autosuficiencia" y "Autonomía"), son aportes que complementan la caracterización de la $\mathrm{AE}$ en Chile, con características compartidas con otras culturas como la anglosajona, pero con matices propios y contextualizados para la realidad cultural chilena.

Estas dos propuestas de estructuras factoriales, esto es, la estructura de cuatro factores de los ítems del IDEA original y la de tres factores correspondiente a los ítems émicos, fueron sometidas a revisión mediante un análisis factorial confirmatorio, técnica que confirmó el modelo unifactorial latente compuesto por seis indicadores, donde se mantuvieron tres indicadores que contenían 21 ítems originales del IDEA y tres indicadores que conservaron 24 ítems émicos, lo que conformó, finalmente, un solo instrumento de 45 ítems que, lo cual no estaba pronosticado. Este resultado confirma la complementariedad entre dimensiones y caracteriza el constructo de AE como una sola etapa de vida, que contiene aspectos éticos y émicos y proporciona un panorama general e integrado de la AE en universitarios chilenos. Por estas razones, relacionadas con la continuidad y complementariedad del constructo de AE, el instrumento final es denominado IDEA - extendido.

En consecuencia, el IDEA - extendido, para Chile, se compone de los siguientes indicadores: "Apertura personal", "Autosuficiencia", "Construcción de la identidad" (conformados por ítems éticos u originales), y "Relaciones interpersonales", "Autonomía" y "Reflexión" (integrados por los ítems émicos). Como se informó, se obtuvieron valores adecuados, tanto para la escala total $(\alpha=0.89)$ como para las seis subescalas que lo componen $(\alpha=$ entre 0.60 y 0.79$)$, con indicadores robustos de bondad de ajuste.

Llama la atención que en el AFC fuese eliminado el indicador de "Presión y estrés", que contenía ítems originales del IDEA relacionados con características ciertamente negativas, como confusión, sentirse restringido, estrés, entre otros aspectos. Su eliminación se debe primeramente a razones psicométricas; sin embargo, es un resultado que no se esperaba, pues existe evidencia teórica y empírica que da cuenta de la alta prevalencia en trastornos de salud mental, específicamente ansiedad, depresión y estrés en esta etapa de vida (Antúnez \& Vinet, 2012; Melchior, Berkman, Niedhammer, Zins, \& Goldberg, 2007; Micin \& Bagladi, 2011). Desde el punto de vista teórico, se podría hipotetizar que los adultos emergentes chilenos de esta muestra no perciben la inestabilidad de la $\mathrm{AE}$ descrita en la conceptualización original de $\mathrm{AE}$, o, a su vez, no perciben de forma tan intensa estos síntomas o malestares que sus pares anglosajones. Otra de las hipótesis que surge es que este hallazgo puede tener relación con que esta dimensión recogía aspectos que no necesariamente tienen relación con estos tres cuadros clínicos, y solo están planteados como síntomas leves o circunstanciales. Sin duda, es necesario 
profundizar estos hallazgos y volver a realizar una revisión exhaustiva de los ítems que están contenidos en esta dimensión.

En cuanto a las fortalezas de este estudio, se destaca la obtención del IDEA - extendido, que es un instrumento de autoinforme adaptado, que tiene adecuados niveles de confiabilidad interna, que mide las características centrales de la $\mathrm{AE}$ en Chile, y en donde sus ítems miden tanto aspectos éticos de la $\mathrm{AE}$ como aspectos émicos o culturales de los jóvenes universitarios chilenos. Así, este instrumento permite captar las particularidades culturales de la AE chilena, que hacen referencia a contenidos relacionados con las "Relaciones interpersonales", la importancia de la "Autonomía" y la importancia de la "Reflexión" como proceso transversal en esta etapa. Asimismo, el IDEA - extendido es un instrumento breve, de fácil aplicación, que está disponible para caracterizar no solo a este grupo de jóvenes, en el ámbito nacional, sino también podría ser aplicado y estudiado en países como Estados Unidos o países con una realidad cultural similar a Chile.

En cuanto a las limitaciones, a pesar de que la teoría de AE abarca diversos grupos de jóvenes de entre 18 y 29 años, esta investigación solo se centró en los universitarios chilenos de universidades públicas, por tanto, los resultados deben limitarse a este grupo de estudio. Se desconoce si la AE está presente en otros grupos de jóvenes chilenos y, de existir en estos otros grupos, si asume características distintivas o no.

Otra limitación consistió en que, al trabajar con universitarios, el rango de edad se vio limitado, pues la media de edad de quienes accedieron a participar fue de 21 años. Por tanto, futuras investigaciones podrían ampliar el rango de edad de adultos emergentes e incluir a grupos de mayor edad que permitan realizar contrastes entre grupos etarios.

Finalmente, es posible señalar que la adultez emergente, además de ser una importante etapa de vida, es una teoría comprensiva del periodo de vida entre 18 y 29 años. Los jóvenes que transitan por este camino se ven enfrentados a cambios, posibilidades, inestabilidad y grandes desafíos. El camino hacia la adultez en estos once largos años es extenso y desafiante, por tanto, para evaluarlo se hace necesario contar con instrumentos validados como el IDEA - extendido, donde se incluyan contenidos émicos y éticos, en pos de identificar y describir fielmente las características de estos jóvenes que transitan esta etapa, un grupo lleno de posibilidades y que tomará las decisiones futuras el día de mañana.

\section{Referencias}

Antúnez, Z., \& Vinet. E. (2012). Escalas de depresión, ansiedad y estrés (DASS - 21): Validación de la Versión Abreviada en Estudiantes Universitarios Chilenos. Terapia Psicológica, 30, 49-55.

Arnett, J. J. (2000). Emerging adulthood: A theory of development from the late teens through the twenties. American Psychologist, $55,469-480$

Arnett, J. J. (2004). Adolescence and emerging adulthood: A cultural approach (2nd ed.). Upper Saddle River, New Jersey: Pearson.

Arnett, J. J. (2012). The Clark University Poll of Emerging Adults. Recuperado de http://www.clarku.edu/clark-poll-emerging-adults/

Arnett, J. J., Kloep, M., Hendry, L., \& Tanner, J. (2011). Debating Emerging Adulthood, Stage or Process? New York: Oxford University Press.

Berry, J. W., Poortinga, Y. H., Segall, M. H., \& Dasen, P. R. (1992). Cross-cultural psychology: Research and applications. Cambridge: Cambridge University Press.

Barrera-Herrera, A. (2016). Caracterización y Evaluación de la Adultez Emergente en Universitarios Chilenos. (Tesis de Doctorado no publicada), Universidad de La Frontera, Temuco, Chile.

Crocetti, E., Tagliabue, S., Sugimura, K., Nelson, L. J., Takahashi, A., Niwa, T., ... Jinno, M. (2015). Perceptions of Emerging Adulthood: A Study with Italian and Japanese University Students and Young Workers. Emerging Adulthood, 3(4), 229-243.

Dutra-Thomé, L. (2013). Emerging Adulthood in southern brazilians from differing socioeconomic status: social and subjective markers. (Tesis de Doctorado) Universidade Federal do Rio Grande do Sul, Brasil. Recuperado desde http://www.lume.ufrgs.br/bitstream/ handle/10183/76534/000886149.pdf?sequence=1

Fierro, D., \& Moreno, A. (2007). Emerging Adulthood in Mexican and Spanish Youth: Theories and Realities. Journal of Adolescent Research, 22, 476-503.

Hu, L. T., \& Bentler, P. M. (1999). Cutoff Criteria for Fit Indexes in Covariance Structure Analysis: Conventional Criteria Versus New Alternatives. Structured Equation Modeling 6, 1-55.

Lisha, N., Grana, R., Sun, P., Rohrbach, L., Spruijt-Metz, D., Reifman, A., \& Sussman, S. (2012). Evaluation of the Psychometric 
Properties of the Revised Inventory of the Dimensions of Emerging Adulthood (IDEA-R) in a Sample of Continuation High School Students. Evaluation \& the Health Professions, 37, 156-177.

Melchior, M., Berkman, L. F., Niedhammer, I., Zins, M., \& Goldberg, M. (2007). The mental health effects of multiple work and family demands: A prospective study of psychiatric sickness absence in the French GAZEL study. Social Psychiatry and Psychiatric Epidemiology, 42, 573-582.

Micin, S., \& Bagladi, V. (2011). Salud Mental en Estudiantes Universitarios: Incidencia de Psicopatología y antecedentes de conducta suicida en población que acude a un Servicio de Salud Estudiantil. Terapia Psicológica, 29, 53-64.

Muñiz, J., Elosua, P., \& Hambleton, R.K. (2013). Directrices para la traducción y adaptación de los test. Psicothema, 25, 149-155.

Pérez, J. C., Cumsille, P., \& Martínez, M. (Marzo, 2008). Construct Validity of the Inventory of Dimensions of Emerging Adulthood in a Chilean Sample. Póster presentado en Society for Research on Adolescence Biennial Meeting, Chicago, Illinions, USA.

Reifman, A., Arnett, J., \& Colwell, M. (2007). Emerging adulthood: Theory, assessment, and application. Journal of Youth Development, 2, 39-50. 